\title{
Quantum control of BEC in optical lattice*
}

\author{
Quan-Fang Wang ${ }^{\dagger}$
}

\begin{abstract}
In this paper, it would be worthwhile to consider the theoretical and computational approach of controlling Bose-Einstein Condensates (BEC). In high spatial dimension (2D/3D) case, the BEC system is controlled under external force in trapped optical lattice at low temperature. Finally, our conclusion is in accordance with the results in physics/chemistry realms.
\end{abstract}

\section{INTRODUCTION}

Since 1990's, one can make the bosonic atoms reach BoseEinstein condensates in real lab experiments (cf. [1], [2], [4], [5]). After that, Bose-Einstein condensates (BEC) always is the focus point in the condensates matter physics last a couple of decades. It has been sufficiently investigated both in physics and chemistry fields.

However, control problems of BEC is merely been considered in quantum control area. With the development of quantum physics field, control Bose Einstein condensates (BEC) become possible in optical trapped lattice at low temperature.

Actually, at the standpoint of physics area, if an ultracold vapor of bosonic atoms are trapped in magnetic well, pure condensates will be created as they are cooled to a temperature below the BEC threshold. After that creation, these BEC are located into a optical lattice potential which can be realized experimentally by a far detuned laser beam. This phenomenon of macroscopic quantum system consisting of ultra-cold atoms in unique in precision and flexibility for experimental control and manipulation.

To push the progress of the field of quantum system control, naturally, Bose Einstein condensates regarded as a quantum control system, and made it changed under the external control forces.

The aim of this paper is to deal with control of BEC in optical lattice with the changing of different laser intense pulse as control input. Our purpose is to deduce the theoretic results and execute numerical simulation demonstration.

Th article is organized as follows. In Section II, BoseEinstein condensates is given by Gross Pitaevskii equation. In Section III, the mathematical setting is presented in Hilbert space. The theoretical conclusion for the BEC quantum system is obtained. In Section IV, computational approach is performed for $4 \times 4$ optical lattice. The simulated graphics are shown in series figures. Lastly, in Section V, resultant conclusion is contained.

\footnotetext{
* Previous work reported on 243rd ACS National Meeting, Poster. San Diego, USA, 2012.

$\dagger$ Mechanical and Automation Engineering, Chinese University of Hong Kong, Shatin, N. T. Hong Kong.
}

\section{BOSE-EINSTEIN CONDENSATES}

Bose-Einstein condensates (BEC) is usually modeled by the celebrated Gross Pitaevskii (GP) equation, a cubically nonlinear Schrödinger equation, see [4].

$$
i \hbar \psi_{t}=-\frac{h^{2}}{2 m}+\mathbf{u}(\mathbf{x}) \psi+N \alpha|\psi|^{2} \psi
$$

where $m$ denote the atomic mass, $\hbar$ is the reduced Planck constant, $N$ is the number of atoms in the condensate, and $\alpha=4 \pi \hbar^{2} / m$, with $a \in R$ denoting the characteristic scattering length of the particles. Here, for simplify to denote

$$
\mathbf{u}(\mathbf{x})=U(\mathbf{x})+V_{0}(\mathbf{x})
$$

where external potential $U(\mathbf{x})$ is confining in order to describe the electromagnetic trap needed for the experimental realization of a BEC. Assumed harmonic form

$$
U(\mathbf{x})=m \omega_{0}^{2} \frac{|\mathbf{x}|^{2}}{2}, \omega_{0} \in R \text { at } t=0 .
$$

A particular example for periodic potentials used in physical experiments is then given by

$$
V_{0}(\mathbf{x})=s \sum_{i=1}^{3} \frac{h^{2} \mathbf{x}^{2}}{m} \sin ^{2}\left(x_{i} x_{i}\right), \quad x_{i} \in R,
$$

where $\mathbf{x}=\left(x_{1}, x_{2}\right)$ denotes the wave vector of the applied laser field and $s>0$ is a dimensionless parameter describing the depth of the optical lattice, which expressed in terms of the recoil energy.

The GP equation (1) provides a case for nonlinear codes since it features high frequency oscillations, two scale external potentials, and (focusing/defocusing) nonlinearity.

\section{QUANTUM CONTROL FOR BEC}

Consider the control problem for BEC described by GP system (1). Firstly, let us regard the issue into mathematical setting in Hilbert space.

Let $\Omega$ be an open bounded set of $R^{2}$ and $Q=(0, T)$ for $T>0$. Then $(\mathbf{x}, t) \in Q$. Introduce two Hilbert spaces

$$
H=L^{2}(\Omega), V=H_{0}^{1}(\Omega)
$$

with usual norm and inner products. Then embedding in Gelfand triple space $V \hookrightarrow H \hookrightarrow V$ are continuous, dense and compact.

Suppose $\mathcal{U}=L^{2}(\Omega)$ is the space of external (e.g. laser optical lattice) controls $\mathbf{u}$. Let $\mathcal{U}_{a d}$ be a closed and convex admissible set of $\mathcal{U}$. Assume initial ground state for each particle in trapped optical lattice as

$$
\psi(\mathbf{u}, 0)=\psi_{0} .
$$


The objective function associated with (1) is given by

$$
J(\mathbf{u})=\epsilon_{1}\left|\psi(\mathbf{u}, T)-\psi_{\text {target }}(\mathbf{u})\right|_{V}+\epsilon_{2}(\mathbf{u}, \mathbf{u}) \mathcal{U} .
$$

Here $\mathbf{u} \in \mathcal{U}_{a d}$, and $\psi_{\text {target }}(\mathbf{u})$ is target state, $\psi(\mathbf{u}, T)$ is observed final states, respectively. Moreover, $\epsilon_{i}, i=1,2$ are weighted coefficients for balancing the values of inherent cost and running cost.

Our goal is to find and characterise quantum optimal control $\mathbf{u}^{*}$ for GP system (1). Here $\mathbf{u}^{*}$ will be called quantum optimal control for GP system (1) subject to objective function (2).

In order to do theoretical control for GP system (1) with objective function (2), first of all, we give two definitions to define the weak solution and its solution space.

Definition 1: Define solution space of the weak solution by Hilbert space (cf. [3]).

$W\left(0, T, V, V^{\prime}\right)=\left\{\psi \mid \psi \in L^{2}(0, T ; V), \psi^{\prime} \in L^{2}\left(0, T ; V^{\prime}\right)\right\}$.

Definition 2: A function $\psi$ is called weak solutions of (1) if $\psi \in W\left(0, T ; V, V^{\prime}\right)$ and satisfy

$$
\begin{aligned}
& \int_{0}^{T} \int_{\Omega} i \hbar \psi_{t} d t d \mathbf{x} \\
= & -\frac{h^{2}}{2 M} \int_{0}^{T} \int_{\Omega} \psi d \mathbf{x} d t+\int_{0}^{T} \int_{\Omega} \mathbf{u} \psi d \mathbf{x} d t \\
& +\int_{0}^{T} \int_{\Omega} N \alpha|\psi|^{2} d \mathbf{x} .
\end{aligned}
$$

By the analogical manipulation as in [6] and take account into (3), it is easy to prove the following two theorems. Let us omit proof in here via the restriction of paper length.

Theorem 3: If given initial state $\psi_{0} \in V$, there exists at least one quantum optimal control $\mathbf{u}^{*}$ for GP system (1) subject to objective function (2).

Theorem 4: For given $\psi_{0} \in V$, there exists at least one quantum optimal control $\mathbf{u}^{*}$ for GP system (1) subject to (2). The optimality systems are given by

$$
\begin{aligned}
& \left\{\begin{array}{l}
i \psi_{t}=-\frac{\hbar^{2}}{2 M} \psi+\mathbf{u}^{*} \psi+N \alpha|\psi|^{2} \psi \\
\psi\left(u^{*}, 0\right)=\psi_{0} \text { in } \Omega
\end{array}\right. \\
& \left\{\begin{array}{l}
i p_{t}=-\frac{\hbar^{2}}{2 M} p+2|\psi| \psi p+|\psi|^{2} p=0 \text { in } Q \\
i p(T)=p\left(\mathbf{u}^{*}, T\right)-\psi_{\text {target }} \text { in } \Omega
\end{array}\right. \\
& \left(\mathbf{u}^{*}, \mathbf{u}-\mathbf{u}^{*}\right) \mathcal{U}+\int_{Q} p\left(\mathbf{u}^{*}\right)\left(\mathbf{u}-\mathbf{u}^{*}\right) d x d t \geq 0, \forall \mathbf{u} \in \mathcal{U}_{a d} \text { (6) }
\end{aligned}
$$

where $p \in W\left(0, T ; V, V^{\prime}\right)$ is the solution of adjoint systems (5) corresponding to $\psi$ in state systems (4).

As is well known the inequality (6) is the necessary optimality condition.

\section{COMPUTATIONAL APPROACH}

In this work, Wang semi-discrete algorithm (cf. [6], [7]) is executed in controlling quantum dynamics (1).

Precisely, finite element approach will be utilized to solve state solution. The updated conjugate gradient method (CGM) for nonlinear case will be used to seek the optimization solution of minimization problem of approximate cost functional consist of (2).
Notice that, at here, the time space is continuous, and the spatial space will be discrete by finite element method (FEM) for meeting the wave-particle duality property for per quantum particle.

\section{A. Experiment setting}

Set $\Omega=[0,15] \times[0,15]$ for two dimensions spatial case. The reduced Planck constant is

$$
\hbar=1.0545715964207855 \times 10^{34} .
$$

The mass of per particle

$$
{ }^{87} R b=1.41923 \times 10^{25} \text { a.u. }
$$

The coefficient in nonlinear term of (1) is $4 \pi \hbar^{2} a / m$, and $a=5.1 \times 10^{9}$.

Let $N=16$ particles are located at lattice, and their coordination calculated through

$$
\begin{aligned}
& x_{i}=3 i, \quad i=1,2,3,4 . \\
& x_{j}=3 j, \quad j=1,2,3,4 .
\end{aligned}
$$

Then we have

$$
\begin{aligned}
& \left(x_{1}, y_{1}\right),\left(x_{1}, y_{2}\right),\left(x_{1}, y_{3}\right),\left(x_{1}, y_{4}\right), \\
& \left(x_{2}, y_{1}\right),\left(x_{2}, y_{2}\right),\left(x_{2}, y_{3}\right),\left(x_{2}, y_{4}\right), \\
& \left(x_{3}, y_{1}\right),\left(x_{3}, y_{2}\right),\left(x_{3}, y_{3}\right),\left(x_{3}, y_{4}\right), \\
& \left(x_{4}, y_{1}\right),\left(x_{4}, y_{2}\right),\left(x_{4}, y_{3}\right),\left(x_{4}, y_{4}\right)
\end{aligned}
$$

respectively. Let $d t=1.0 \times 10^{4}$ and the iteration $n$ number $n=5, T=n d t$.

To configure each state function, the appendix function is given by

$$
\begin{aligned}
\Phi(\mathbf{x}, v, t)= & \frac{1}{1.2247448713915894} \frac{3 \sqrt{2}}{4 \sqrt{1-v^{2}}}\left(\sec \left[\frac{(\mathbf{x}-v t)}{2 \sqrt{1-v^{2}}}\right]\right)^{2} \\
& \exp \left[i\left(v \mathbf{x}+\frac{1-v^{2}+v^{4}}{2\left(1-v^{2}\right)} t\right)\right]
\end{aligned}
$$

where $v$ are adjustable parameters, and take $v=\frac{5}{6}$ in demonstration of Section IV. Therefore, the initial state of each particles is given by

$$
\psi_{0}=\Phi(\mathbf{x}, v, 0) .
$$

Take the target state of each particles by

$$
\psi_{\text {target }}=\Psi(\mathbf{x}, v, T)
$$

The initial control input (i.e. optical lattice configuration) is supposed by

$$
\mathbf{u}_{0}(t)=1.0 \times 10^{19} \sin \left(1.5 \times 10^{4} t\right) .
$$

The initial control variable $\mathbf{u}_{0}(t)$ is plotted in Figure 1 . 


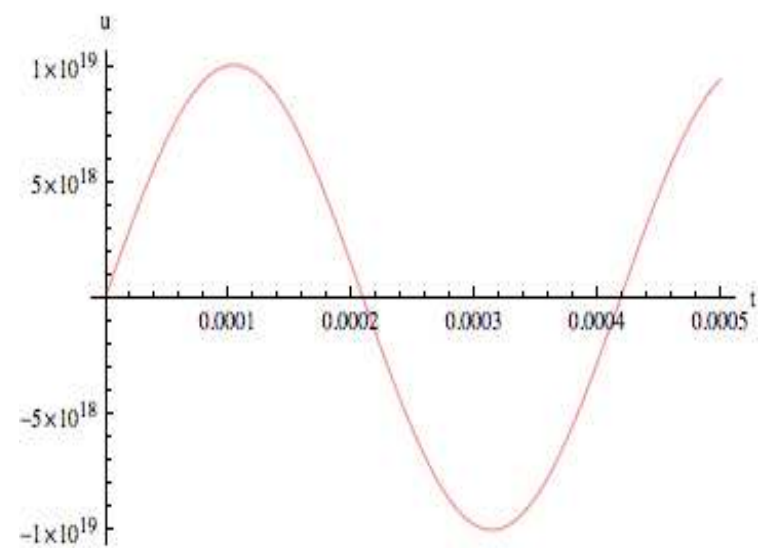

FIG. $1 \mathbf{u}_{0}(t)$ for $t \in[0,0.0005]$. (red curve)

Take the external potential as

$V(x, y, \lambda)=V_{0} \exp \sum_{k=1}^{N} \exp \left(-\frac{\lambda^{2}\left(x-x_{j}\right)^{2}+\left(y-y_{j}\right)^{2}}{2 \omega^{2}}\right)$.

Here $V_{0}=-0.6, \omega=1.0$ are given parameters. Take $\lambda=\frac{\mathbf{u}}{T}$ at each iteration in subsection $B$.

\section{B. Experiment demonstration}

By the above experiment setting in subsection $A$ of Section IV. It is well for us to proceed the simulating demonstration with the Mathematica 7. Particularly, 'Visual Quantum Mechanics' (VQM) package is used for graphics expression of BEC described by GP system (1).

For initial state $\psi_{0}$ given in subsection $A$, its graphics are shown in Figures 2.

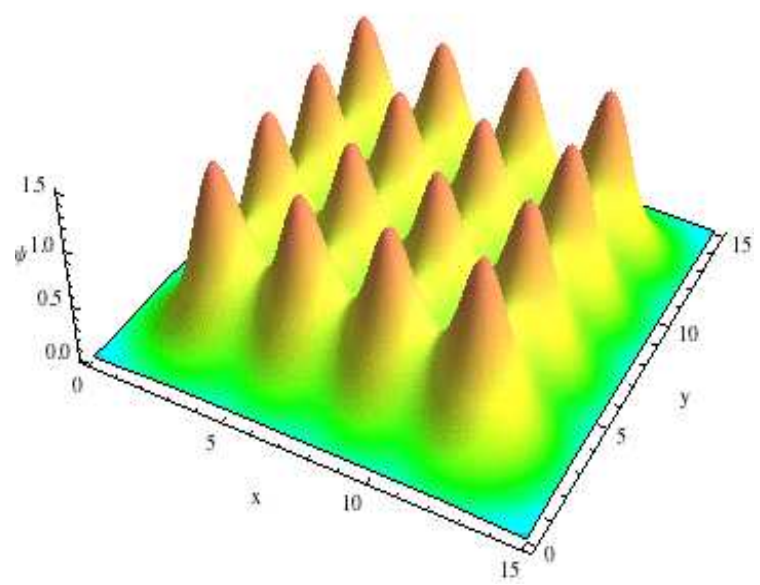

(a). Plot of $\psi_{0}$.

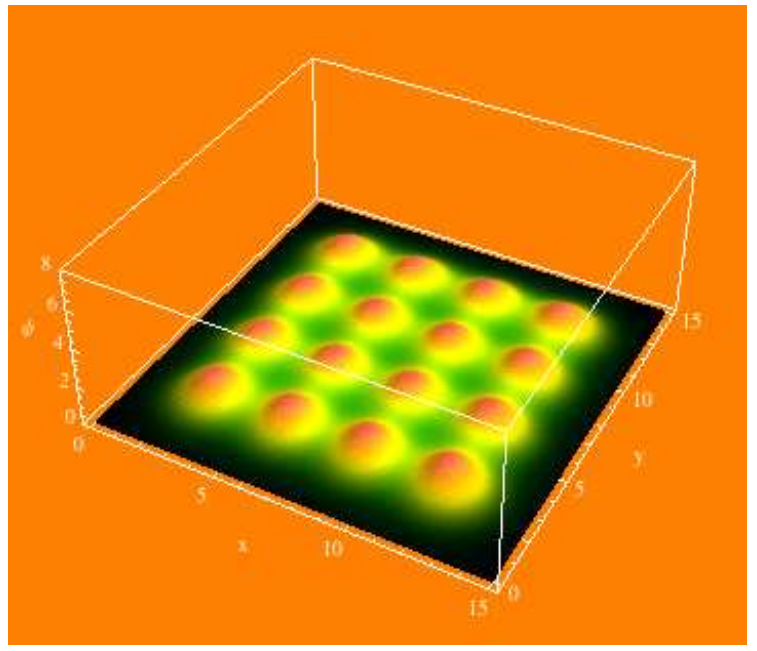

(b). Box plot of $\psi_{0}$.

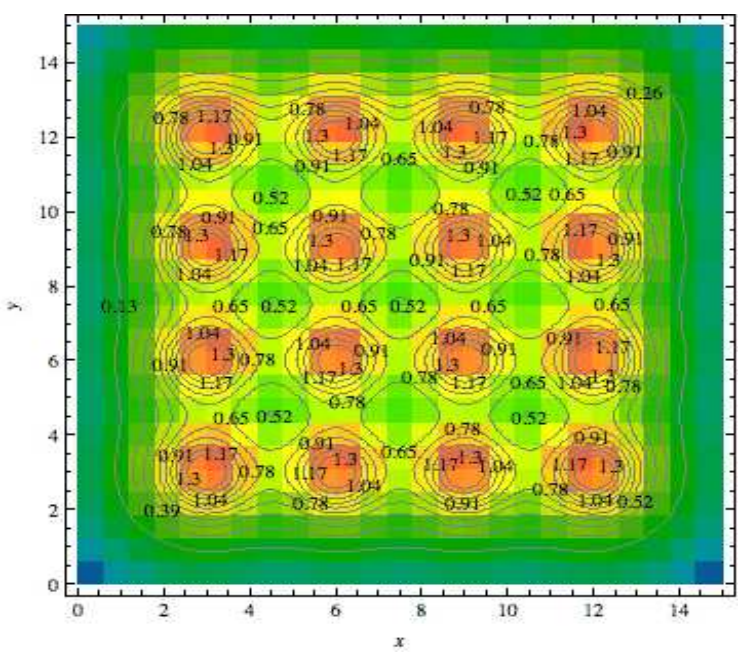

(c). Contour plot of $\psi_{0}$.

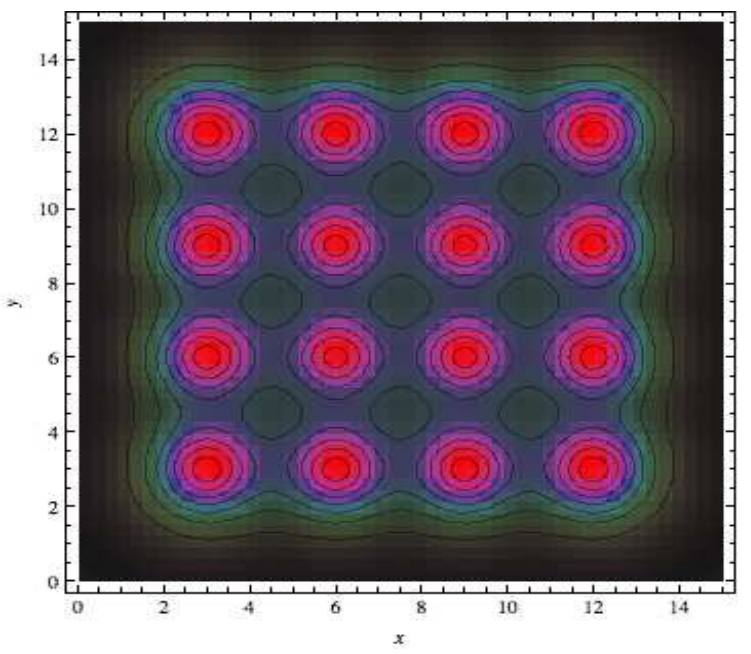

(d). Black white contour of $\psi_{0}$.

FIG. 2 Plot and contour plot $\psi(0), \mathbf{x} \in[0,15] \times[0,15]$.

For target state $\psi_{\text {target }}$ at time $T$, its graphics are computed in Figure 3. 


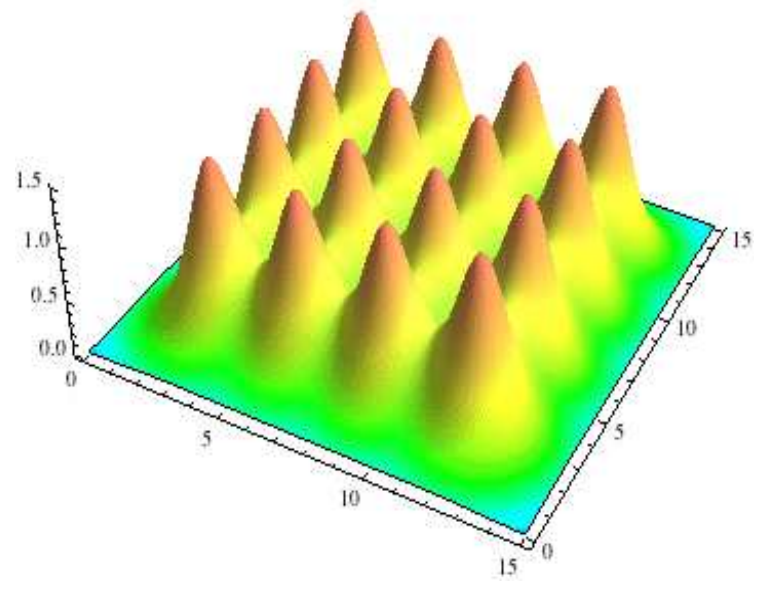

(a). Plot of $\psi_{\text {target }}$.

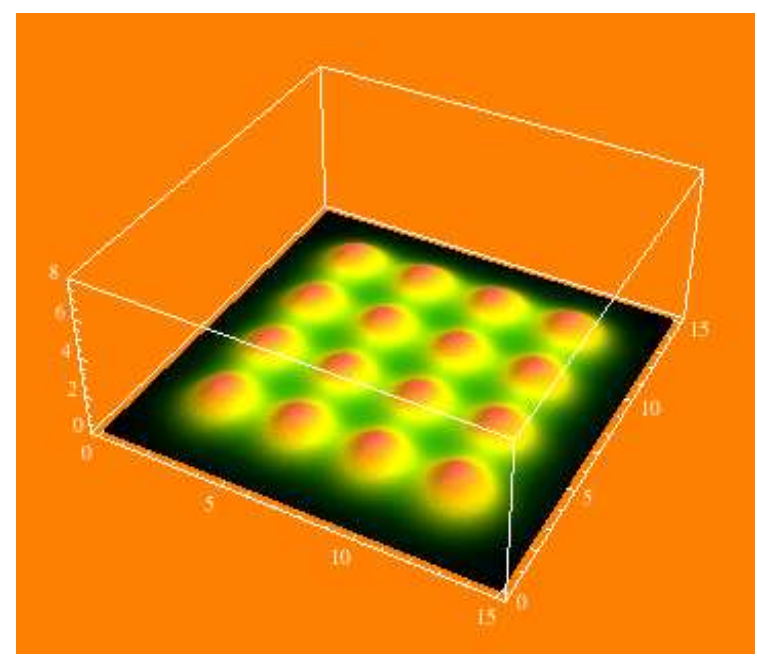

(b). Box plot of $\psi_{\text {target }}$.

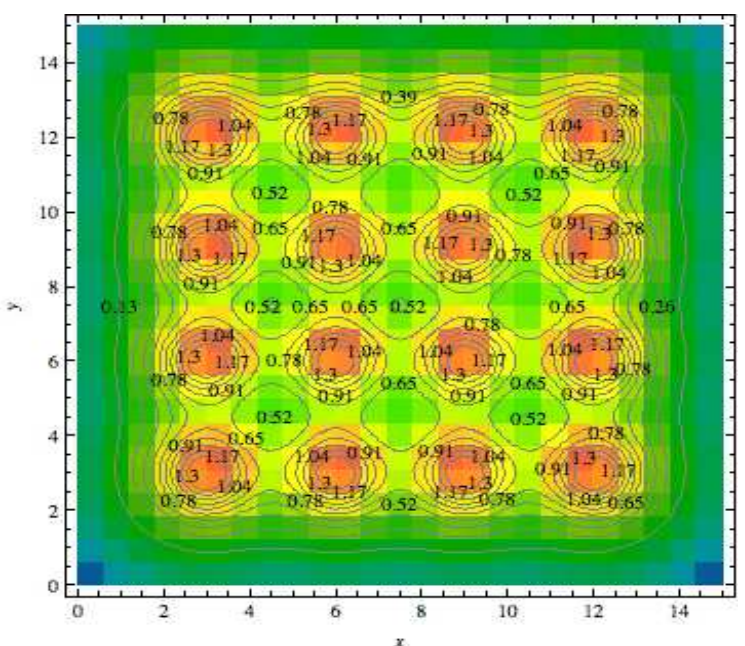

(c). Contour plot of $\psi_{\text {target }}$.

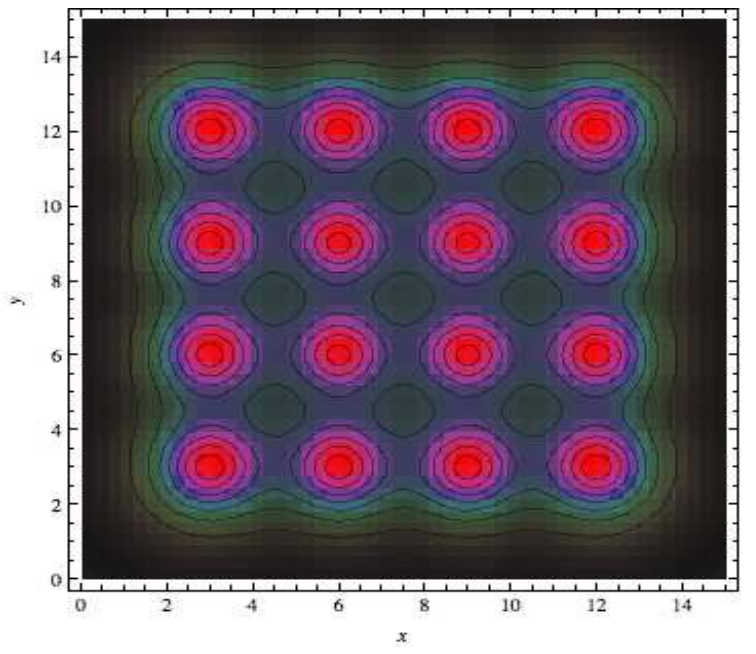

(d). Black white contour of $\psi_{\text {target }}$.

FIG. 3 Plot and contour plot $\psi(T), \mathbf{x} \in[0,15] \times[0,15]$.

After running serval round iterations, at $n=5$, the calculated states are shown in Figure 4.

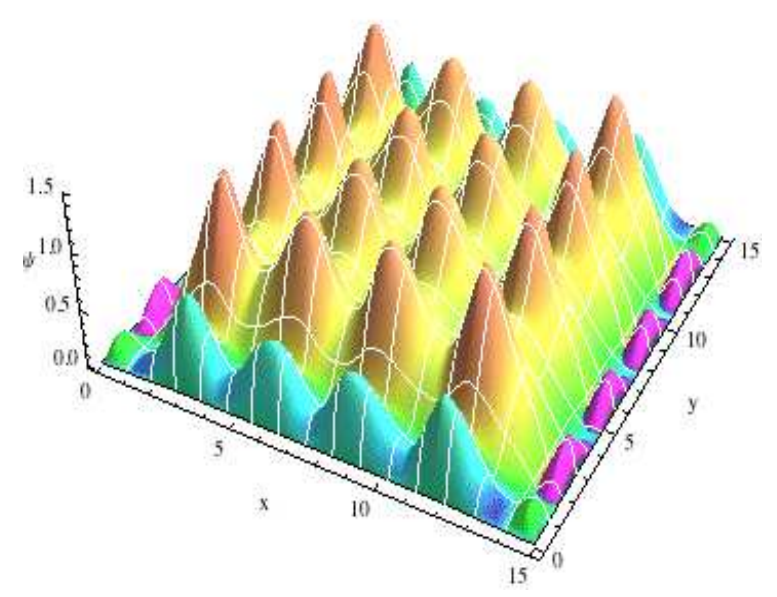

(a). Plot of $\psi_{n}$.

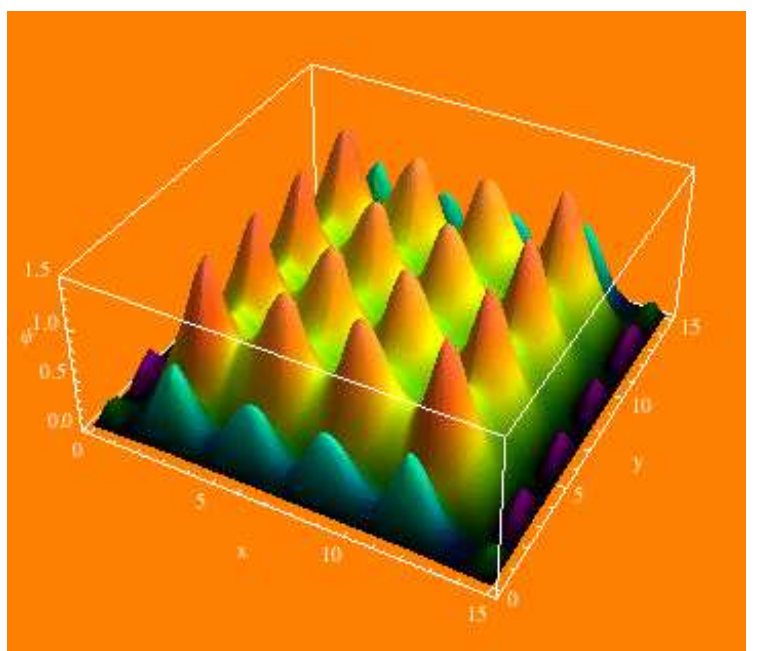

(b). Box plot of $p s i_{n}$. 


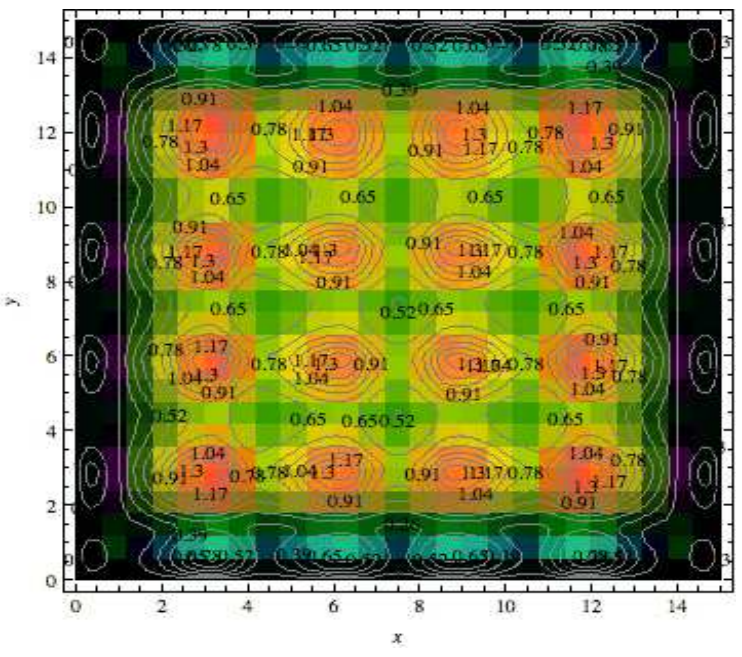

(c). Contour plot of $\psi_{n}$.

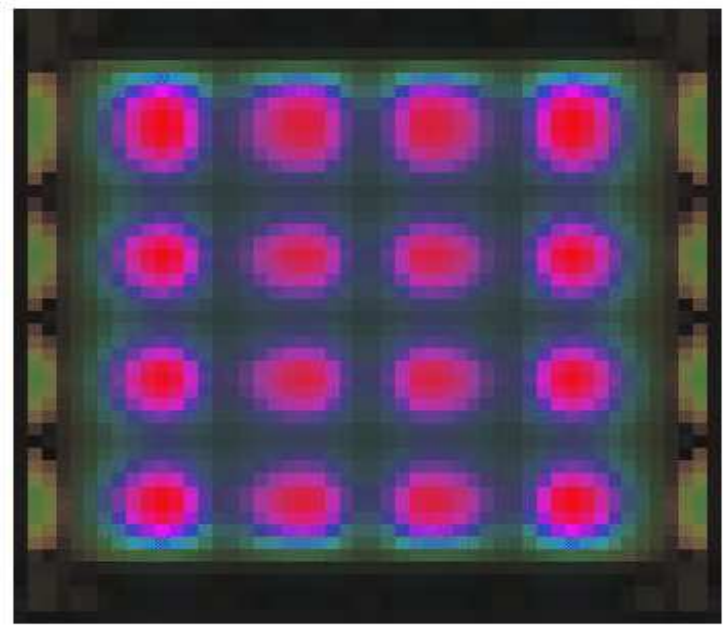

(d). Black white contour of $\psi_{n}$.

FIG. 4 Plot and contour plot of $\psi(\mathbf{u}, t)$ at $n=5$, $\mathbf{x} \in[0,15] \times[0,15]$.

Adjoint system are also calculated and plotted in Figure 5 at iteration $n=5$.

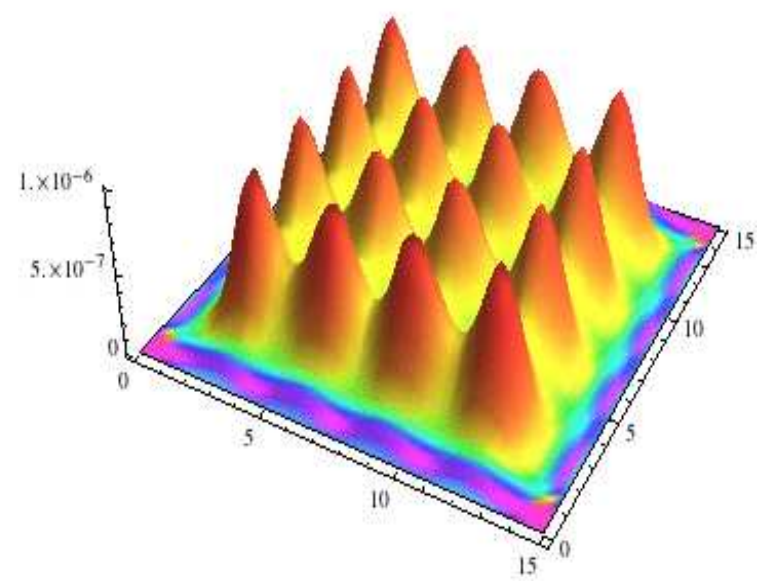

(a). Plot of $p(\mathbf{u}, t)$.

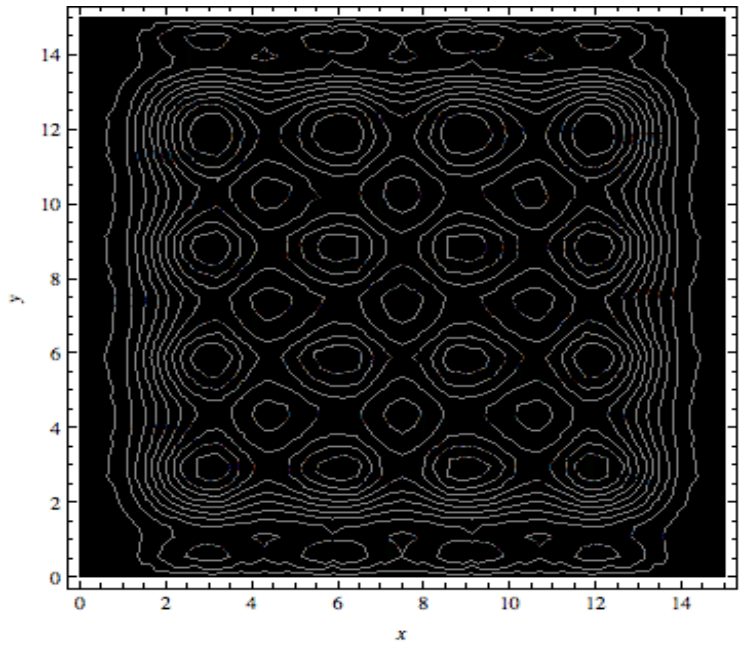

(b). Contour plot of $p(\mathbf{u}, t)$

FIG. 5 Plot and contour plot of adjoint state $p(\mathbf{u}, t)$, $\mathbf{x} \in[0,15] \times[0,15]$.

Quantum optimal control lattice express as (iteration $n=4$ )

$$
\mathbf{u}^{*}=\left(-8.41477 \times 10^{40}+1.0 \times 10^{19} \sin (15000.0 t)\right) \text {. }
$$

Its graphics is plotted in Figures 6.

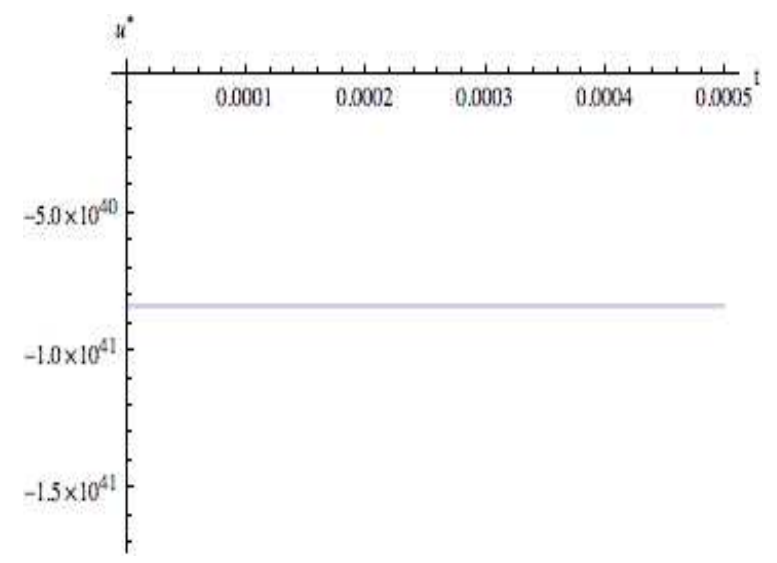

FIG. $6 \mathbf{u}^{*}(t)$ for $t \in[0,0.0005]$. (almost a line around $-8.41477 \times 10^{40}$ )

Optimal cost function value are computed at $n=4$ as

$$
J\left(\mathbf{u}^{*}\right)=3.38171 \times 10^{63} .
$$

Furthermore, the cost functions iterations $J(\mathbf{u})$ with unit a.u. at each iteration is displayed in Figures 7. 


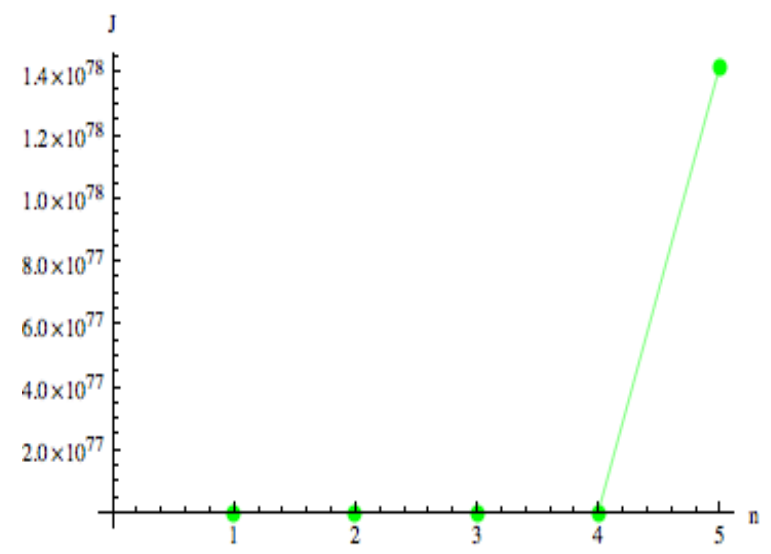

FIG. $7 J\left(\mathbf{u}_{n}\right), n=1,2,3,4,5$ (green dots)

Also, the error values of cost functions $e J=J\left(\mathbf{u}_{n+1}\right)-$ $J\left(\mathbf{u}_{n}\right)$ at each iteration refer to Figures 8.

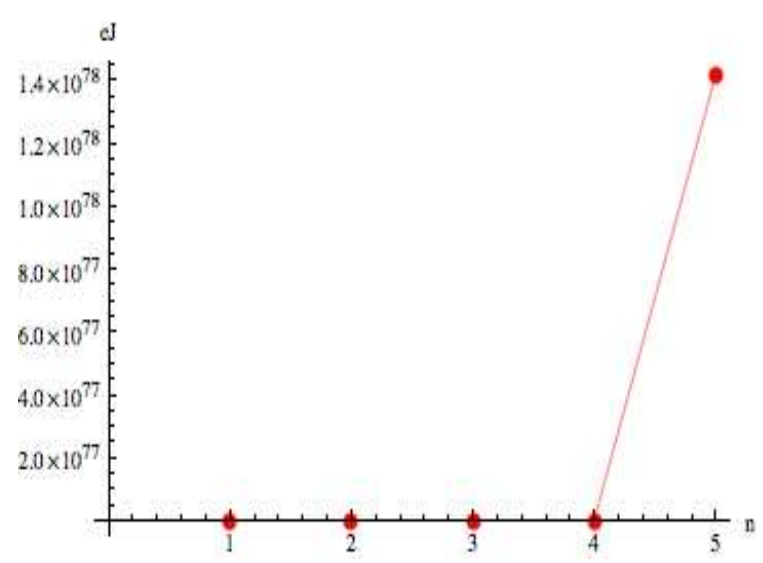

FIG. 8 Error $e J, n=1,2,3,4,5$ (red dots)

Moreover, the evaluate of cost functions can be compared in Table I.

TABLE I

COST VALUeS AT EACH ITERATION

\begin{tabular}{ll}
\hline iteration & cost $J$ \\
\hline $\mathrm{n}=1$ & $1.04663 \times 10^{34}$ \\
\hline $\mathrm{n}=2$ & $1.00205 \times 10^{47}$ \\
\hline $\mathrm{n}=3$ & $3.38171 \times 10^{63}$ \\
\hline $\mathrm{n}=4$ & $3.38171 \times 10^{63}$ \\
\hline $\mathrm{n}=5$ & $1.41617 \times 10^{78}$.
\end{tabular}

The error values at each iteration are listed in Table II too.

Obviously, one can found that the particles are reached condensate status in the iteration $n=4$ at the $4 \times 4$ optical lattice. At this moment, there is no more external energy is needed, and the status is holding for a while as condensate state for each particle.

Definitely, the simulated results are agree with the literature reported for BEC in chemistry and physics fields (cf. [1], [2], [4], [5], [9]).

The total used CUP time is 4001.96 second, and the occupied memory is 254805008 bytes.
TABLE II

ERROR VALUES AT EACH ITERATION

\begin{tabular}{lll}
\hline iteration & error cost $e J$ & \\
\hline $\mathrm{n}=1$ & $1.04663 \times 10^{34}$ & $* e J\left(\mathbf{u}_{1}\right)=J\left(\mathbf{u}_{1}\right)$ \\
\hline $\mathrm{n}=2$ & $1.00205 \times 10^{47}$ & \\
\hline $\mathrm{n}=3$ & $3.38171 \times 10^{63}$ \\
\hline $\mathrm{n}=4$ & 0 \\
\hline $\mathrm{n}=5$ & $1.1617 \times 10^{78}$.
\end{tabular}

\section{RESULTS AND DISCUSSION}

In summary, the controlling for BEC have been solved regarding the quantum dynamics to seek the optimal solution (cf. [9]). This study extremely acquire the real laboratory evidence for quantum controlling achievement. The progress would be a promising research direction (cf. [6], [7], [8], [9]).

Future research would be interested in focus on the controlling of three dimension (3D) Bose-Einstein condensates (BEC) at optical lattice. It is left to our further publications. With the aiding of computer science development, the computational approach and simulation would become true for high dimension study of controlling BEC as well as physical phenomena in quantum mechanics. As a bright perspective of quantum system control in present and future, it can be considered as possible and feasible.

It is a attempt work of BEC and would be valuable as constant surveys for a long time.

\section{ACKNOWLEDGMENTS}

The author thanks to the 243rd ACS National Meeting \& Exposition for poster entitled "Quantum control for BoseEinstein condensates in theory and numerical experiment" (cf. [9]), and also thanks to Prof. V. P. Belavkin (The University of Nottingham, United Kingdom) for co-works.

\section{REFERENCES}

[1] A. Bulatov, B. E. Vugmeister and H. A. Rabitz, Nonadiabatic control of Bose-Einstein condensation in optical traps, Physical Review A 60(6), 1998, pp 4875-4881.

[2] J. Grond, et al, Optimal control of trapped Bose-Einstein condensates, Physical Review A 80, 2009, p. 053625.

[3] J. L. Lions, Optimal Control of Systems Governed by Partial Differential Equations, Berlin-Heidelberg-New York, Springer-Verlag, 1971.

[4] L. Pitaevskii and S. Stringari, Bose-Einstein Condensation, Internat. Ser. Monogr. Phys. 116, Clarendon Press, Oxford, UK, 2003.

[5] S. A. Rice et al, Optical Control of Molecular Dynamics, New York, Wiley, 2000.

[6] Q. F. Wang, Quantum optimal control of nonlinear dynamics systems described by Klein-Gordon-Schrödinger equations, Proceeding of American Control Conference, 2006, pp 1032-1037.

[7] Q. F. Wang and H. A. Rabitz, Quantum optimal control for the KleinGordon-Schrödinger dynamics system in the presence of disturbances and uncertainties, Gordon Research Conference "Quantum Control of Light and Matter", 2007, poster.

[8] Q. F. Wang, C. Cao, Quantum numerical optimal control of nucleon and meson dissipative dynamical model, Proceeding of the European Control Conference, 2009, pp 168-172.

[9] Q. F. Wang, Quantum control for Bose-Einstein condensates in theory and numerical experiment, 243rd ACS National Meeting \& Exposition, 2012, (Poster). 